\title{
THE DISPERSION OF THE COEFFICIENTS OF UNIVALENT FUNCTIONS
}

BY

\author{
D. H. HAMILTON ${ }^{1}$
}

\begin{abstract}
The Hayman $T_{a}$ function for the asymptotic distribution of the coefficients of univalent functions has a continuous derivative which is closely related to the asymptotic behavior of coefficient differences.
\end{abstract}

1. Introduction. Suppose that $S$ denotes the class of functions $f(z)=z+$ $\sum_{m=2}^{\infty} a_{m} z^{m}$ which are univalent in the disk $\{|z|<1\}$. We define

$$
A_{n}=\sup \left\{\left|a_{n}\right|: f \in S\right\} \text {. }
$$

FitzGerald's method (see Horowitz [9]) shows that $A_{n} \leqslant(1.05 \ldots) n$, and Hayman [7] showed that $A_{n} / n \rightarrow K_{0}$, where $K_{0}$ is some absolute constant. (However it is not even known if $A_{n}$ is increasing.) A closely related result is Nehari's [11] proof that

$$
\left|a_{n}\right| \leqslant 4 n K_{0} \operatorname{dist}(0, \mathrm{C}-f(|z|<1)),
$$

which was extended by Bombieri [1]. Recently FitzGerald [3] showed that if $f_{n}(z)=\sum_{m=1}^{\infty} a_{n, m} z^{m}$ in $S$ has $\left|a_{n, n}\right| \sim A_{n}$ then $\left|a_{n, 2}\right| \rightarrow 2$. Hamilton [5] gave a simpler proof of a more general result and noted that this implies Littlewood's conjecture is equivalent to Hayman's, i.e., $\left|a_{n}\right| \leqslant 4 n \operatorname{dist}(0, \mathbf{C}-f(|z|<1))$ for all $f \in S \Rightarrow K_{0}=1$. (See $[4,5,6]$ for related results.)

This paper is concerned with extending asymptotic results of this type. Hayman [7] proves that if $f_{n}=\sum_{m=1}^{\infty} a_{n, m} z^{m}$ is a sequence in $S$ such that

$$
\lim _{n \rightarrow \infty} n^{-2}\left|f_{n}\left(1-\frac{1}{n}\right)\right|=\lambda>0 \text {, }
$$

then $a_{n, m} / n$ converges on a subsequence of $n \rightarrow \infty$ and as $m / n \rightarrow a>0$ to a continuous function $T_{a}$. We prove that $T_{a}$ has continuous derivative and as $n \rightarrow \infty$, $m / n \rightarrow a>0$,

$$
a_{n, m+1}-a_{n, m} \rightarrow T_{a}^{\prime} .
$$

This result has a number of interesting consequences.

THEOREM 1. Suppose that $f_{n} \in S$ and $a_{n, n}=A_{n}$; then as $n \rightarrow \infty$,

$$
\left|a_{n, m+1}\right|-\left|a_{n, m}\right| \rightarrow K_{0} \text {, }
$$

provided that $m / n \sim 1$.

Received by the editors November 18, 1981 and, in revised form, March 9, 1982.

AMS (MOS) subject classifications (1970). Primary 30C55.

${ }^{\mathrm{I}}$ The author was partially supported by NSF grant number 81.0380 . 
Remark 1. We deduce from (3) and the Marty relation yet another proof of $a_{n, 2} \rightarrow 2$. Also we prove

THEOREM 2. As $n \rightarrow \infty$,

$$
A_{n+1}-A_{n} \rightarrow K_{0} .
$$

REMARK 2. In particular $A_{n}$ is an increasing sequence for large $n$.

REMARK 3. Relation (4) also shows that there is no essential difference between the odd and even coefficients, as was found by Bombieri [2] near the Koebe function.

We begin with a discussion of Hayman's asymptotic theory, concluding that section with a statement of some technical results. The following sections are devoted to proving these statements. Finally we obtain Theorems 1 and 2.

2. $H$-theory. We consider subsequences

$$
f_{n}(z)=z+\sum_{m=2}^{\infty} a_{n, m} z^{m}
$$

which satisfy (2). Then some subsequence of $n^{-2} f_{n}(1-z / n)$ converges locally uniformly on the half plane $\{\operatorname{Re}(z)>0\}$ to a function $\phi(z)$ such that:

$$
\begin{gathered}
\phi(z) \text { is nonzero on }\{\operatorname{Re}(z)>0\}, \\
\phi(z) \text { is univalent on }\{\operatorname{Re}(z)>0\}, \\
\lim _{x \rightarrow \infty} x^{2}|\phi(x)|=\alpha \leqslant 1 .
\end{gathered}
$$

The class $H$ consists of those functions $\phi$ which satisfy properties (7)-(9). Furthermore for each $\phi \in H$ there is a sequence $f_{n}$ in $S$, satisfying (2), such that

$$
n^{-2} f_{n}(1-z / n) \rightarrow \phi(z) \text { as } n \rightarrow \infty .
$$

Hayman also shows that

$$
\int_{-\infty}^{\infty}|\phi(x+i y)| d y \leqslant \frac{1}{2 x}
$$

for $\phi \in H, x>0$. In particular the Fourier transform

$$
T_{a}(\phi)=\frac{1}{2 \pi} \int_{-\infty}^{\infty} \phi(x+i y) e^{a(x+i y)} d y
$$

exists as a continuous function of $a$. Hayman's main result is

TheOREM A. If $n^{-2} f_{n}(1-z / n) \rightarrow \phi(z) \in H$ as $n \rightarrow \infty$, then $a_{n, m} / n \rightarrow T_{a}(\phi)$ as $n \rightarrow \infty$ and $m / n \rightarrow a>0$.

Consequently the problem of bounding the linear functional $T_{1}(\phi)$ on $H$ is equivalent to finding $\varlimsup_{n \rightarrow \infty} A_{n} / n$, i.e., $K_{0}=\sup \left\{\left|T_{1}(\phi)\right|: \phi \in H\right\}$. The main result of this paper is

THEOREM 3. For each $\phi \in H$ and positive $a$, the improper integral

$$
V_{a}(\phi)=\frac{1}{2 \pi} \int_{-\infty}^{\infty}(x+i y) \phi(x+i y) e^{a(x+i y)} d y
$$


exists as a continuous function of a. Furthermore if $f_{n} \in S$ and $n^{-2} f_{n}(1-z / n) \rightarrow \phi(z)$ then

$$
a_{n, m+1}-a_{n, m} \rightarrow V_{a}(\phi)
$$

as $m / n \rightarrow$ a and $n \rightarrow \infty$.

REMARK 4. For fixed $x$, the function $(x+i y) \phi(x+i y)$ is not an $L_{1}$ function of $y$ (as compared with (10)). Thus Theorem 3 is established in an indirect manner.

EXAmPLE 1. Suppose we choose

$$
f_{n}(z)=\frac{z}{\left(1-2 z \cos (t / n)+z^{2}\right)} .
$$

Then it is not hard to show that

$$
n^{-2} f_{n}(1-z / 2) \rightarrow 1 /(z+t)^{2} .
$$

Thus $T_{a}=\sin (a t) / t, T_{a}^{\prime}=\cos (a t)$, while

$$
a_{n, m+1}-a_{n, m}=\frac{\sin \{(m+1) t / n\}-\sin \{m t / n\}}{\sin (t / n)} \rightarrow \cos (a t)
$$

as $n \rightarrow \infty$ and $m / n \rightarrow a$.

Corollary 1. $T_{a}(\phi)$ has continuous derivative on $(0, \infty)$.

EXAMPLE 2. In [5] it was shown how $H$-theory easily implies Hayman's results on functions $f \in S$ such that

$$
r^{-1}(1-r)^{2}|f(r)| \rightarrow \alpha>0
$$

For then putting $f_{n}=f$ we find that

$$
n^{-2} f_{n}(1-z / n) \rightarrow \alpha e^{i \theta} / z^{2}=\phi(z)
$$

along some subsequence, where different $\theta$ may arise from different subsequences. Thus $T_{a}(\phi)=\alpha e^{i \theta} a$ and $T_{a}^{\prime}(\phi)=\alpha e^{i \theta}$. Consequently by Theorem $3,\left|a_{n+1}\right|-\left|a_{n}\right|$ $\rightarrow \alpha$, which was the second result in [8].

3. Preliminary results. As we are assuming (2) we need to know the location of points of maximum modulus of $f\left(r e^{i \theta}\right)$ on $\{|z|=r\}$. Let $M(r, f)=\max _{\theta}\left|f\left(r e^{i \theta}\right)\right|$.

Lemma 1. Suppose that $f_{n} \in S$ satisfies (2). Then for any positive $x$ the maximum modulus of $f_{n}$ on $\{|z|=1-x / n\}$ occurs at a point of argument $\eta(x)$ which satisfies for large $n$

$$
\eta(x) \leqslant A(\lambda, x) / n
$$

where $A(\lambda, x)$ is a finite constant depending on $\lambda$ and $x$ only. ${ }^{2}$

Suppose that $n^{-2}\left|f_{n}(1-x / n)\right| \rightarrow 0$ on some subsequence. Then the corresponding limit function $\phi(z) \in H$ satisfies $\phi(x)=0$, which is impossible. Thus as $n \rightarrow \infty$,

$$
n^{-2}\left|f_{n}(1-x / n)\right| \geqslant A(x, \lambda)>0 .
$$

We frequently use the following inequality of Hayman [7, p. 11].

\footnotetext{
${ }^{2}$ The symbols $A, A(\cdot, \cdot)$, etc. will be used to denote constants which depend on parameters shown.
} 
Lemma A. Suppose that $f \in S$ satisfies $M(r, f)=|f(r)| \geqslant \lambda /(1-r)^{2}, \lambda>0$. Then

$$
\left|f\left(r e^{i \theta}\right)\right| \leqslant \frac{A(\lambda, \varepsilon)}{(1-r)^{2-\varepsilon}|\theta|^{\varepsilon}}
$$

for any $0<\varepsilon<2$.

We apply Lemma A to $e^{-i \eta} f_{n}\left(e^{i \eta} z\right), r=1-x / n$ and $\varepsilon=1$, noting

$$
M\left(1-x / n, f_{n}\right) \geqslant A(\lambda, x)(1-(1-x / n))^{-2},
$$

to obtain

$$
\left|f_{n}(1-x / n)\right| \leqslant A n^{2} /|\eta|
$$

which contradicts (15) unless (14) holds.

In general we want to consider the expression $a_{n, m}-e^{i t / n} a_{n, m-1}$. We shall assume that $t=0$ and $M\left((1-1 / m), f_{n}\right)=\left|f_{n}(1-1 / m)\right|$. Suppose that we have established Theorem 3 under this assumption. Now for general $f_{n}$ satisfying (2), Lemma 1 shows that the maximum of $\left|f\left((1-1 / m) e^{i \theta}\right)\right|$ occurs at a point having argument $\eta$, $|\eta| \leqslant A(\lambda, x) / n, x=n / m \sim 1 / a$. Thus the function $e^{-i \eta} f_{n}\left(e^{i \eta} z\right)$ satisfies condition (2) and has maximum modulus at $(1-1 / m)$. Consequently

$$
a_{n, m} e^{i(m-1) \eta}-a_{n, m-1} e^{i(m-2) \eta} \rightarrow \frac{1}{2 \pi} \int_{-\infty}^{\infty}(x+i y) \phi(x+i(y-\nu)) e^{a(x+i y)} d y
$$

as if $n^{-2} f_{n}(1-z / n) \rightarrow \phi(z)$; then by Lemma 1

$$
n^{-2} e^{-i \eta} f_{n}\left(e^{i \eta}(1-z / n)\right) \rightarrow \phi(x+i(y-\nu))
$$

where $\eta n \rightarrow \nu, z=x+i y$. Changing the variable and noting that $e^{i(m-1) \eta} \rightarrow$ $e^{i(m / n) \eta n} \rightarrow e^{i a \nu}$ gives

$$
e^{i a \nu}\left(a_{n, m}-e^{i(-\nu) / n} a_{n, m-1}\right) \rightarrow \frac{e^{i a \nu}}{2 \pi} \int_{-\infty}^{\infty}(x+i(y+\nu)) \phi(x+i y) e^{a(x+i y)} d y .
$$

Now by Theorem A,

$$
\frac{a_{n, m-1}}{n} \rightarrow \frac{1}{2 \pi} \int_{-\infty}^{\infty} \phi(x+i y) e^{a(x+i y)} d y=T_{a},
$$

and consequently (16) becomes

$$
a_{n, m}-e^{-i \nu / n} a_{n, m-1} \rightarrow \frac{1}{2 \pi} \int_{-\infty}^{\infty}(x+i y) \phi(x+i y) e^{a(x+i y)} d y+i \nu T_{a} .
$$

Thus we obtain $a_{n, m}-a_{n, m-1} \rightarrow V_{a}$. For general $t$ we have

$$
a_{n, m}-e^{i t / n} a_{n, m-1} \rightarrow V_{a}+i t T_{a} .
$$

Thus we have shown that to obtain (17) it suffices to assume the maximum of $\left|f_{n}\left((1-1 / m) e^{i \theta}\right)\right|$ occurs at $(1-1 / m)$.

4. $a_{n, m}-a_{n, m-1}$ remains bounded. To show $a_{n, m}-a_{n, m-1}$ remains bounded we need modifications of Hayman's argument. These modified formulae and Hayman's lemmas will be needed to prove Theorem 3.

Lemma 2. Suppose that $f \in S$ satisfies $M(1-1 / m, f)=|f(1-1 / m)|$. Then $\left|a_{m}-a_{m-1}\right| \leqslant A$, where $A$ is an absolute constant. 
Following Hayman we write

$$
(m-1) a_{m-1}-\left(1-\frac{1}{m}\right) m a_{m}=\frac{1}{2 n i} \int_{|z|=\rho} \frac{(z-(1-1 / m))}{z^{m}} f^{\prime}(z) d z .
$$

Next we integrate (18) with respect to $\rho$ from $1-3 / m$ to $1-2 / m$. The left-hand side of $(18)$ becomes $((m-1) / m)\left\{a_{m-1}-a_{m}\right\}$, while the right-hand side becomes

$$
\frac{1}{2 \pi} \int_{1-3 / m}^{1-2 / m} \int_{|z|=\rho} \frac{e^{-i(m-1) \theta}}{\rho^{m}}\left(\rho e^{i \theta}-\left(1-\frac{1}{m}\right)\right) f^{\prime}\left(\rho e^{i \theta}\right) \rho d \theta d \rho .
$$

Consequently

$$
a_{m-1}-a_{m}=\frac{m}{m-1}\left\{I_{1}+I_{2}\right\}
$$

where for $j=1,2$ we have

$$
I_{j}=\frac{1}{2 \pi} \iint_{\mathscr{D}_{j}} \frac{e^{-i(m-1) \theta}}{\rho^{m}}\left(\rho e^{i \theta}-\left(1-\frac{1}{m}\right)\right) f^{\prime}\left(\rho e^{i \theta}\right) \rho d \theta d \rho,
$$

and $\mathscr{D}_{1}=\left\{z: 1-3 / m \leqslant|z| \leqslant 1-2 / m, \delta_{2} / n \leqslant \operatorname{Arg}(z) \leqslant \delta_{1} / n\right.$, for some $\delta_{2}<0$ $\left.<\delta_{1}\right\}$, and $\mathscr{D}_{2}=\{1-3 / m \leqslant|z| \leqslant 1-2 / m\}-\mathscr{D}_{1}$. Now let us define $M_{1}=$ $M(1-1 / m, f)$ and regions $\varepsilon_{k} \subset \mathscr{Q}_{1} \cup \mathscr{Q}_{2}$ by

$$
\varepsilon_{k}: 2^{-k} M_{1} \leqslant|f(z)| \leqslant 2^{1-k} M_{1} .
$$

The following lemma of Hayman [8, p. 238] is useful.

LEMMia B. If $M_{k}=2^{1-k} M_{1}$ and $G_{k}(R)=M_{k}^{2} R^{2} /\left(M_{k}^{2}+R^{2}\right)$, then for $1-3 / m \leqslant$ $\rho \leqslant 1-2 / m$,

$$
\int_{0}^{2 \pi}\left|\rho e^{i \theta}-\left(1-\frac{1}{m}\right)\right|^{2} G_{k}\left(\left|f\left(\rho e^{i \theta}\right)\right|\right) d \theta \leqslant A m 2^{-k / 4},
$$

where $A$ is an absolute constant.

Now returning to $(20)$ we have

$$
\left|I_{j}\right| \leqslant A \iint_{\mathscr{D}_{j}}\left|\rho e^{i \theta}-\left(1-\frac{1}{m}\right)\right| f^{\prime}\left(\rho e^{i \theta}\right) \mid \rho d \theta d \theta
$$

as $\rho^{-m} \leqslant e^{-3}$. Consequently

$$
\begin{aligned}
\left|I_{j}\right| \leqslant & A \sum_{k=1}^{\infty}\left(\int_{\mathscr{D}_{j} \cap \varepsilon_{k}}\left|\frac{f^{\prime}(z)}{f(z)}\right|^{2} \rho d \rho d \theta\right)^{1 / 2} \\
& \times\left(\iint_{\mathscr{D}_{j} \cap \varepsilon_{k}}\left|z-\left(1-\frac{1}{m}\right)\right|^{2}|f(z)|^{2} d \theta \rho d \rho\right)^{1 / 2} .
\end{aligned}
$$

Now

$$
\begin{aligned}
\iint_{\varepsilon_{k}}\left|\frac{f^{\prime}(z)}{f(z)}\right|^{2} \rho d \rho d \theta & \leqslant M_{k+1}^{-2} \iint_{\varepsilon_{k}}\left|f^{\prime}(z)\right|^{2} \rho d \rho d \theta \\
& \leqslant M_{k+1}^{-2} \operatorname{Area}\left(f\left(\varepsilon_{k}\right)\right) \leqslant M_{k+1}^{-2} \pi M_{k}^{2}=4 \pi .
\end{aligned}
$$


On the other hand, for $z \in \varepsilon_{k}$,

$$
|f(z)|^{2} \leqslant \frac{2 M_{k}^{2}|f(z)|^{2}}{M_{k}^{2}+|f(z)|^{2}} .
$$

Thus

$$
\left|I_{j}\right| \leqslant A \sum_{k=1}^{\infty}\left(\int_{\mathcal{D}_{j} \cap \varepsilon_{k}}\left|z-\left(1-\frac{1}{m}\right)\right|^{2} G_{k}(|f(z)|) \rho d \rho d \theta\right)^{1 / 2} .
$$

Thus by Lemma B,

$$
\left|I_{j}\right| \leqslant A \sum_{k=1}^{\infty}\left(\int_{1-3 / m}^{1-2 / m} A m 2^{-k / 4} \rho d \rho\right)^{1 / 2} \leqslant A,
$$

which by (19) completes the proof of Lemma 2.

5. Estimating $I_{2}$. The formulae of $\S 4$ enable us to estimate $I_{2}$. Let $\delta=\min \left(\delta_{1},-\delta_{2}\right)$ (see (21)).

LEMMA 3. With $f_{n}$ satisfying (2) and $I_{2}$ defined by (20), $m / n \sim a>0$,

$$
\left|I_{2}\right| \leqslant A(\lambda, a) \delta^{-1 / 8} \text {. }
$$

Now the definition of $\mathscr{D}_{2}$ and Lemma $A$, with $\varepsilon=1$, implies that for $|\theta| \geqslant \delta / n$, $1-3 / m \leqslant \rho \leqslant 1-2 / m$,

$$
\left|f\left(\rho e^{i \theta}\right)\right| \leqslant A(\lambda, a) m^{2} / \delta
$$

where we used Lemma 1 to ensure that $|f(\rho)| \geqslant A(\lambda, a) /(1-\rho)^{2}$ in the range $1-3 / m \leqslant \rho \leqslant 1-2 / m, m / n \sim a$. Thus for

$$
k<\left[\left(\log ^{+}(\delta)+\log ^{+} A(\lambda, a)\right) / \log (2)\right]=k_{0},
$$

$\varepsilon_{k} \cap \mathscr{D}_{2}=\varnothing$. Consequently by (23),

$$
I_{2} \leqslant A \sum_{k=k_{0}}^{\infty}\left(\iint_{D_{2}}\left|z-\left(1-\frac{1}{m}\right)\right| G_{k}\left(\left|f_{n}(z)\right|\right) \rho d \rho d \theta\right)^{1 / 2} \leqslant A 2^{-k_{0} / 8}
$$

by Lemma B. Thus by (25) and (26), $I_{2} \leqslant A(a, \lambda) \delta^{-1 / 8}$ which proves Lemma 2 .

6. Estimating $I_{1}$. To obtain asymptotic expressions for $I_{1}$ we take a sequence $f_{n} \in S$ such that $n^{-2} f_{n}(1-z / n) \rightarrow \phi(z)$. Then we write $f_{n}=f$, and use the results of $\S \S 4$ and 5. Integrating by parts,

$$
\begin{aligned}
\int_{\delta_{2} / n}^{\delta_{1} / n} \frac{e^{-i(m-1) \theta}}{\rho^{m}}\left(\rho e^{i \theta}-\right. & \left.\left(1-\frac{1}{m}\right)\right) f^{\prime}\left(\rho e^{i \theta}\right) d \theta \\
= & \int_{\delta_{2} / n}^{\delta_{1} / n} \frac{(-i)}{\rho^{m}} e^{-i m \theta}\left(\rho e^{i \theta}-\left(1-\frac{1}{m}\right)\right) f\left(\rho e^{i \theta}\right) \\
& +\int_{\delta_{2} / n}^{\delta_{1} / n} \frac{(m-1)}{\rho^{m}}\left\{\rho e^{-i(m-1) \theta}-e^{-i m \theta} f\left(\rho e^{i \theta}\right)\right\} d \theta
\end{aligned}
$$


where the two terms of the right-hand side are denoted by $J_{1}$ and $J_{2}$, respectively, and

$$
I_{1}=\frac{1}{2 \pi} \int_{1-3 / m}^{1-2 / m}\left(J_{1}+J_{2}\right) d \rho .
$$

LEMMA 4. With the above assumptions,

$$
\left|\int_{1-3 / m}^{1-2 / m} J_{1} d \rho\right| \leqslant \frac{A(a, \lambda, \varepsilon)}{\delta^{1-\varepsilon}},
$$

where $\delta=\min \left(\delta_{1},-\delta_{2}\right)>2, m / n \sim a>0$.

Now

$$
\begin{aligned}
& \left|\int_{1-3 / m}^{1-2 / m} \frac{e^{-i(m / n) \delta_{j}}}{\rho^{m}}\left(\rho e^{i \delta_{j} / n}-\left(1-\frac{1}{m}\right)\right) f\left(\rho e^{i \delta_{j} / n}\right) d \rho\right| \\
& \quad \leqslant A n^{-1} \max _{1-3 / m \leqslant \rho \leqslant 1-2 / m}\left|f\left(\rho e^{i \delta_{j} / n}\right)\right|_{1-3 / m \leqslant \rho \leqslant 1-2 / m}\left|\rho e^{i \delta_{j} / n}-\left(1-\frac{1}{m}\right)\right|,
\end{aligned}
$$

using $\rho^{-m} \leqslant e^{3}, m / n \sim a$. By Lemmas 1 and $\mathrm{B}$,

$$
\left|f\left(\rho e^{i \delta_{j} / n}\right)\right| \leqslant \frac{A(a, \lambda, \varepsilon)}{\delta_{j}^{2-\varepsilon}} n^{2}
$$

and

$$
\begin{aligned}
\max _{1-3 / m \leqslant \rho \leqslant 1-2 / m}\left|\rho e^{i \delta_{j} / n}-\left(1-\frac{1}{m}\right)\right| & \leqslant A\left|\left(1-\frac{3}{m}\right)\left(1+\frac{i \delta_{j}}{n}\right)-\left(1-\frac{1}{m}\right)\right| \\
& =A\left|\frac{2}{m}-\frac{i \delta_{j}}{n}\right| \leqslant \frac{A}{n} \delta_{j},
\end{aligned}
$$

provided $\delta_{j} \geqslant 2$. Substituting (30) and (31) in (29) gives

$$
\left|\int_{1-3 / m}^{1-2 / m} J_{1} d \rho\right| \leqslant \sum_{j=1}^{2} \frac{A}{\delta_{j}^{1-\varepsilon}}<\frac{A}{\delta^{1-\varepsilon}},
$$

as required.

Next we estimate the second term in (28).

Lemma 5. Suppose that $n^{-2} f_{n}(1-(x+i y) / n) \rightarrow \phi(x+i y) \in H$ as $n \rightarrow \infty$. Then

$$
\begin{aligned}
\frac{1}{2 \pi} \int_{1-3 / m}^{1-2 / m} \int_{\delta_{2} / n}^{\delta_{1} / n} \frac{(m-1) e^{-i m \theta}}{m-1} & \left\{\rho e^{i \theta}-1\right\} f_{n}\left(e^{i \theta}\right) \rho d \theta d \rho \\
= & -\frac{1}{2 \pi} \int_{\delta_{2}}^{\delta_{1}}(x+i y) \phi(x+i y) e^{a(x+i y)} d y+o(1),
\end{aligned}
$$


as $m / n \rightarrow a>0$ and $n \rightarrow \infty$. The error term is bounded by

$$
\begin{gathered}
A\left(\delta_{1}, \delta_{2}, a\right)\left\{\left|\frac{m}{n}-a\right|+\frac{1}{n}+\max _{\delta_{2} \leqslant y \leqslant \delta_{1}}\left|\frac{f_{n}}{n^{2}}\left(\left(1-\frac{x}{n}\right) e^{-i y / n}\right)-\phi(x+i y)\right|\right\} \\
+A(a, x, \lambda, \varepsilon) \delta^{\varepsilon-1} .
\end{gathered}
$$

New variables $\rho=1-x / n$ and $\theta=-y / n$ are substituted into the double integral. This becomes

$$
\begin{aligned}
\frac{1}{2 \pi} \int_{2 n / m}^{3 n / m} \int_{\delta_{2}}^{\delta_{1}}(m-1)\left(1-\frac{x}{n}\right)^{-n(m / n) x} e^{i(m / n) y}\left\{\left(1-\frac{x}{n}\right) e^{-i y / n}-1\right\} & \\
& \times f_{n}\left(\left(1-\frac{x}{n}\right) e^{-i y / n}\right) \frac{d x d y}{n^{2}},
\end{aligned}
$$

which is

$$
-\frac{1}{2 \pi} \int_{2 / a}^{3 / a} \int_{\delta_{2}}^{\delta_{1}} a e^{a x} e^{i a y}\{x+i y\} \frac{f_{n}\left((1-x / n) e^{-i y / n}\right)}{n^{2}} d x d y+\varepsilon_{1},
$$

where the error term satisfies

$$
\begin{aligned}
\varepsilon_{1} & <A \int_{2 / a}^{3 / a} \int_{\delta_{2}}^{\delta_{1}} a\left|\left(\frac{m}{n}-a\right)+\frac{1}{n}\right| e^{a x}|x+i y| \frac{\left|f_{n}\right|}{n^{2}} d x d y \\
& <A \max \left(\delta_{1},-\delta_{2}, \frac{3}{a}\right)\left|\frac{m}{n}-a\right|+\frac{1}{n} \int_{\delta_{2}}^{\delta_{1}} \frac{\left|f_{n}\right|}{n^{2}} d x \\
& <A \max \left(\delta_{1},-\delta_{2}, \frac{3}{a}\right)\left\{\left|\frac{m}{n}-a\right|+\frac{1}{n}\right\} .
\end{aligned}
$$

Thus the double integral is equal to

$$
-\frac{a}{2 \pi} \int_{2 / a}^{3 / a} \int_{\delta_{2}}^{\delta_{1}}(x+i y) e^{a(x+i y)} \phi(x+i y) d x d y+\varepsilon_{1}+\varepsilon_{2},
$$

with the second error term satisfying

$$
\varepsilon_{2}<A\left(\max \left(\delta_{1},-\delta_{2}, \frac{3}{a}\right)\right)^{2} \max _{\delta_{2} \leqslant y \leqslant \delta_{1}}\left\{\left|\frac{f_{n}\left((1-x / n) e^{-i y / n}\right)}{n^{2}}-\phi(x+i y)\right|\right\} .
$$

Finally we show that integrating with respect to $x$ in (32) is redundant. From Cauchy's theorem for any $x_{1} \leqslant x_{2}$,

$$
\int_{\delta_{2}}^{\delta_{1}}\left(x_{1}+i y\right) \phi\left(x_{1}+i y\right) e^{a\left(x_{1}+i y\right)} d y=\int_{\delta_{2}}^{\delta_{1}}\left(x_{2}+i y\right) \phi\left(x_{2}+i y\right) e^{a\left(x_{2}+i y\right)} d y+\varepsilon_{3},
$$

where

$$
\begin{aligned}
\varepsilon_{3} & <\sum_{j=1}^{2}\left|\int_{x_{1}}^{x_{2}}\left(x+i \delta_{j}\right) \phi\left(x+i \delta_{j}\right) e^{a\left(x+i \delta_{j}\right)} d x\right| \\
& <\sum_{j=1}^{2}\left(x_{2}-x_{1}\right)\left(x_{2}+\left|\delta_{j}\right|\right) e^{a x_{2}} \max _{x_{1} \leqslant x \leqslant x_{2}}\left|\phi\left(x+i \delta_{j}\right)\right| .
\end{aligned}
$$


However by Lemmas 1 and B, letting $n^{-2} f_{n} \rightarrow \phi$,

$$
\max _{x_{1} \leqslant x \leqslant x_{2}}\left|\phi\left(x+i \delta_{j}\right)\right| \leqslant \frac{A\left(\lambda, x_{1}, x_{2}, \varepsilon\right)}{\delta_{j}^{2-\varepsilon}} .
$$

Thus

$$
\varepsilon_{3}<A\left(x_{1}, x_{2}, \lambda, \varepsilon, a\right) / \delta^{1-\varepsilon}
$$

provided $\delta_{1}, \delta_{2} \geqslant 1$. Combining these results proves the lemma.

7. The proof of Theorem 3. From Lemmas 5 and 6, substituting into (28) we have

$$
I_{1}=-\frac{1}{2 \pi} \int_{\delta_{2}}^{\delta_{1}}(x+i y) \phi(x+i y) e^{a(x+i y)} d y+\varepsilon_{4}+\varepsilon_{5}
$$

where $\varepsilon_{4}<A(a, x, \lambda, \varepsilon) / \delta^{1-\varepsilon}$, and $\varepsilon_{5} \rightarrow 0$ as $n \rightarrow \infty$. Thus by Lemma 3 and (19), we have shown

LEMMA 6. With the above notation

$$
a_{n, m}-a_{n, m-1}=\frac{1}{2 \pi} \int_{\delta_{2}}^{\delta_{1}}(x+i y) \phi(x+i y) e^{a(x+i y)} d y+\varepsilon_{6}+\varepsilon_{7},
$$

where $\varepsilon_{6}<A(a, x, \lambda, \varepsilon) / \delta^{1 / 8}$ and $\varepsilon_{7} \rightarrow 0$ as $n \rightarrow \infty$.

Now in Lemma 2 it was shown that $\left|a_{m}-a_{m-1}\right|$ is bounded. Let $n=n_{q}$ be a subsequence such that

$$
\lim _{q \rightarrow \infty} a_{n_{q}, m+1}-a_{n_{q}, m} \rightarrow V,
$$

where the subsequence will also depend on how $m / n \rightarrow a$. (The limit $V$ may possibly depend on the subsequence.) Then by Lemma 6,

$$
V=\frac{1}{2 \pi} \int_{-\delta_{2}}^{\delta_{1}}(x+i y) \phi(x+i y) e^{a(x+i y)} d y+\varepsilon_{6}+\varepsilon_{7},
$$

where $\varepsilon_{6} \rightarrow 0$ as $\delta \rightarrow \infty$, and $\varepsilon_{7} \rightarrow 0$ as $n \rightarrow \infty$. This proves that the improper integral exists. Thus by Lemma 6 every convergent subsequence of $a_{n, m+1}-a_{n, m}$ converges to the same limit once we have checked that $V_{a}$ is a continuous function of $a$.

LEMMA 7. $V_{a}$ is a continuous function of $a$.

For any $b$ near $a$ by (34)

$$
V-V_{b}=\frac{1}{2 \pi} \int_{\delta_{2}}^{\delta_{1}}(x+i y) \phi(x+i y)\left\{e^{a(x+i y)}-e^{b(x+i y)}\right\} d y+\varepsilon_{8},
$$

where $\varepsilon_{8} \rightarrow 0$ as $\delta \rightarrow \infty$. Thus

$$
\left|V-V_{b}\right| \leqslant A\left(\delta_{1}, \delta_{2}, x\right)|a-b|+\varepsilon_{8},
$$

which shows that $V_{b} \rightarrow V$ as $b \rightarrow a$. This completes the proof of Lemma 8 and consequently of Theorem 3 . The last comments in $\$ 3$ mean that we have also proved (17). 
8. Proof of Corollary 1. This result is proved by showing that $T_{a}^{\prime}(\phi)=V_{a}$, and then by appealing to Theorem 3. Now let $n^{-2} f_{n}(1-z / n) \rightarrow \phi(z)$ as $n \rightarrow \infty$ and $1 / c<a<b<c$. If $m, p$ are integers such that $m / n \rightarrow a, p / n \rightarrow b$ then

$$
\frac{T_{a}-T_{b}}{(a-b)}=\frac{a_{n, m} / n-a_{n, p} / n+\varepsilon_{1}}{m / n-p / n+\varepsilon_{1}},
$$

where $\varepsilon_{1} \rightarrow 0$, uniformly in $c$, as $n \rightarrow \infty$. Now by Theorem 3 ,

$$
a_{n, m}=a_{n, p}+(m-p) V_{a}+(m-p) \varepsilon_{2},
$$

where $\left|\varepsilon_{2}\right|<\varepsilon_{3}+\varepsilon_{4}, \varepsilon_{3} \rightarrow 0$ as $a \rightarrow b$ and $\varepsilon_{4} \rightarrow 0$ as $n \rightarrow \infty$. Thus by (35) and (36)

$$
\frac{T_{a}-T_{b}}{a-b}=\frac{((m-p) / n)\left(V_{a}+\varepsilon_{2}\right)+\varepsilon_{1}}{(m-p) / n+\varepsilon_{1}} \rightarrow V_{a}+\varepsilon_{5},
$$

as $n \rightarrow \infty$ and $\left|\varepsilon_{5}\right|<\varepsilon_{3}$. Thus as $a \rightarrow b,\left(T_{a}-T_{b}\right) /(a-b) \rightarrow V_{a}$, which proves Corollary 1 .

\section{Proof of Theorem 1.}

LEMma 9. Suppose that $\phi(z) \in H$ and $f_{n} \in S$ such that $n^{-2} f_{n}(1-z / n) \rightarrow \phi(z)$ as $n \rightarrow \infty$. Then the limit of $\left(\left|a_{n, m+1}\right|-\left|a_{n, m}\right|\right)_{n \rightarrow \infty}, m / n \rightarrow a$ exists as a continuous function of $a$, provided $T_{a} \neq 0$,

$$
a_{n, m+1}-e^{i t / n} a_{n, m} \rightarrow V_{a}-i t T_{a} .
$$

Thus

$$
|| a_{n, m+1}|-| a_{n, m}|| \rightarrow \min _{-\infty<t<\infty}\left|V_{a}-i t T_{a}\right|,
$$

and the sign of the limit of $\left|a_{n, m+1}\right|-\left|a_{n, m}\right|$ is determined by which side of the line $V_{a}-i t T_{a}$ contains 0 . Notice that at points $a$ such that $T_{a}=0$ we can have a discontinuity (see Example 1). Also $\left|a_{n, m+1}\right|-\left|a_{n, m}\right| \rightarrow 0$ if and only if $V_{a} \perp T_{a}$. Similarly to Corollary 1 we obtain

Corollary 2. $\left|T_{a}\right|$ is a $C^{1}$ function of $a$ on $\left\{a: T_{a} \neq 0\right\}$. For these points $a$, if $m / n \rightarrow$ a as $n \rightarrow \infty$ we have $\left|a_{n, m+1}\right|-\left|a_{n, m}\right| \rightarrow d\left|T_{a}\right| / d a$.

We can now prove Theorem 1. Let $f_{n}$ be a function in $S$ such that $\left|a_{n, n}\right|=A_{n}$. It is not clear that the maximum of $\left|f_{n}\left((1-1 / n) e^{i \theta}\right)\right|$ occurs near $(1-1 / n)$ so we consider

$$
f_{n}^{*}(z)=e^{-i \sigma} f_{n}\left(e^{i \sigma} z\right),
$$

where $\left|f_{n}\left((1-1 / n) e^{i \sigma(n)}\right)\right|=M\left(1-1 / n, f_{n}\right)$. The standard method (see Hayman [7, p. 3]) shows that

$$
\lim _{n \rightarrow \infty} n^{-2} M\left(1-\frac{1}{n}, f_{n}\right)=\lambda>0,
$$

and thus we may apply our analysis to $f_{n}^{*}$. Thus for any subsequence of $n$ such that $n^{-2} f_{n}^{*}(1-z / n) \rightarrow \phi(z)$, for some $\phi(z) \in H$, by Corollary $2,\left|a_{n, m+1}\right|-\left|a_{n, m}\right| \rightarrow$ $d\left|T_{a}(\phi)\right| / d a$ as $n \rightarrow \infty$ along the same subsequence. Also $\left|a_{n, n} / n\right|=A_{n} / n \rightarrow K_{0}$. 
Thus $\left|T_{1}(\phi)\right|=K_{0}$. Then we prove

Lemma 10. Suppose that $\left|T_{1}(\phi)\right|=K_{0}$. Then $d\left|T_{1}(\phi)\right| / d a=K_{0}$.

As $K_{0}=\varlimsup\left(A_{n} / n\right), \varlimsup\left|a_{n, m} / n\right| \leqslant K_{0}(m / n) \rightarrow K_{0} a$ and thus $\left|T_{a}(\phi)\right| \leqslant K_{0} a$ for all $a$. However $\left|T_{1}(\phi)\right|=K_{0}$ and $\left|T_{a}(\phi)\right|$ is smooth near 1 (by Corollary 2), which implies that

$$
\frac{d}{d a}\left|T_{a}\right|_{a=1}=K_{0}
$$

Thus we have shown that any convergent subsequence of $\left|a_{n, m+1}\right|-\left|a_{n, m}\right|$ converges to $K_{0}$ as $n \rightarrow \infty, m / n \rightarrow 1$. This proves the theorem.

REMARK 5. Lucas [10] shows that

$$
|| a_{n+2}|-2| a_{n+1}|+| a_{n}|| \leqslant A n^{1-\sqrt{2}}
$$

but this is not strong enough to prove Theorem 1 .

10. Proof of Theorem 2. Suppose that $f_{n}$ is a subsequence in $S$ with $\left|a_{n, n}\right|=A_{n}$. Then by Theorem 1 we have

$$
\left|a_{n, n+1}\right|-A_{n} \rightarrow K_{0}
$$

and

$$
A_{n+1}-\left|a_{n+1, n}\right| \rightarrow K_{0}
$$

as $n \rightarrow \infty$.

Since $\left|a_{n, n+1}\right| \leqslant A_{n+1}$ and $\left|a_{n+1, n}\right| \leqslant A_{n}$ we deduce

$$
\liminf _{n \rightarrow \infty}\left(A_{n+1}-A_{n}\right) \geqslant K_{0}
$$

from the first limit relation and

$$
\limsup _{n \rightarrow \infty}\left(A_{n+1}-A_{n}\right) \leqslant K_{0}
$$

from the second limit relation. This proves Theorem 2 .

\section{REFERENCES}

1. E. Bombieri, On functions which are regular and univalent in a half plane, Proc. London Math. Soc. (3) 14A (1965), 47-50.

2. On the local maximum property of the Koebe function, Invent. Math. 4 (1967), 26-67.

3. C. H. FitzGerald, Large late coefficients of univalent functions (to appear).

4. D. H. Hamilton, The successive coefficients of univalent functions, J. London Math. Soc. (to appear).

5. __ On a conjecture of Littlewood, Proc. Amer. Math. Soc. (to appear).

6. The equivalence of two weak Bieberbach conjectures, Math. Z. (to appear).

7. W. K. Hayman, Bounds for the large coefficients of univalent functions, Ann. Acad. Sci. Fenn. Ser. AI Math. 250 (1958).

8. __ On successive coefficients of univalent functions, J. London Math. Soc. 38 (1963), 228-243.

9. D. Horowitz (to appear).

10. K. W. Lucas, On successive coefficients of really mean p-valent functions, J. London Math. Soc. 44 (1969), 631-642.

11. Z. Nehari, On the coefficients of univalent functions, Proc. Amer. Math. Soc. 8 (1957), 291-293.

Department of Mathematics, University of California At San Diego, la Jolla, California 92093 